\title{
MRI-based treatment plan simulation and adaptation for ion radiotherapy using a classification-based approach
}

\author{
Christopher M Rank ${ }^{1 *}$, Christoph Tremmel ${ }^{1}$, Nora Hünemohr ${ }^{1}$, Armin M Nagel2 \\ Oliver Jäkel ${ }^{1,3}$ and Steffen Greilich ${ }^{1}$
}

\begin{abstract}
Background: In order to benefit from the highly conformal irradiation of tumors in ion radiotherapy, sophisticated treatment planning and simulation are required. The purpose of this study was to investigate the potential of MRI for ion radiotherapy treatment plan simulation and adaptation using a classification-based approach.
\end{abstract}

Methods: Firstly, a voxelwise tissue classification was applied to derive pseudo CT numbers from MR images using up to 8 contrasts. Appropriate MR sequences and parameters were evaluated in cross-validation studies of three phantoms. Secondly, ion radiotherapy treatment plans were optimized using both MRI-based pseudo CT and reference $\mathrm{CT}$ and recalculated on reference $\mathrm{CT}$. Finally, a target shift was simulated and a treatment plan adapted to the shift was optimized on a pseudo CT and compared to reference $\mathrm{CT}$ optimizations without plan adaptation.

Results: The derivation of pseudo CT values led to mean absolute errors in the range of 81 - $95 \mathrm{HU}$. Most significant deviations appeared at borders between air and different tissue classes and originated from partial volume effects. Simulations of ion radiotherapy treatment plans using pseudo CT for optimization revealed only small underdosages in distal regions of a target volume with deviations of the mean dose of PTV between $1.4-3.1 \%$ compared to reference CT optimizations. A plan adapted to the target volume shift and optimized on the pseudo CT exhibited a comparable target dose coverage as a non-adapted plan optimized on a reference $C T$.

Conclusions: We were able to show that a MRI-based derivation of pseudo CT values using a purely statistical classification approach is feasible although no physical relationship exists. Large errors appeared at compact bone classes and came from an imperfect distinction of bones and other tissue types in MRI. In simulations of treatment plans, it was demonstrated that these deviations are comparable to uncertainties of a target volume shift of $2 \mathrm{~mm}$ in two directions indicating that especially applications for adaptive ion radiotherapy are interesting.

Keywords: Magnetic resonance imaging, lon radiotherapy, lon beam therapy, Treatment planning, Simulation, Plan adaptation, Pseudo CT, Classification, Ultrashort echo time

\section{Background}

Ion radiotherapy offers the opportunity of highly conformal irradiation of tumors applying high doses to the tumor volume while sparing the surrounding tissue and organs at risk [1]. In order to achieve this benefit, sophisticated treatment planning and simulation are required. Major uncertainties in the ion radiotherapy treatment

*Correspondence: c.rank@dkfz.de

${ }^{1}$ German Cancer Research Center (DKFZ), Division of Medical Physics in Radiation Oncology, Im Neuenheimer Feld 280, 69120 Heidelberg, Germany Full list of author information is available at the end of the article process are interfractional and intrafractional changes in patient anatomy, for instance organ and tumor movement, tumor shrinkage, filling of air cavities and loss of weight. Today, the gold standard for radiotherapy treatment plan simulation is Computed Tomography (CT). A stoichiometric Hounsfield look-up table (HLUT) is used to derive water equivalent path length (WEPL) values for ions from the measured X-ray CT numbers.

Here we investigate the potential of Magnetic Resonance Imaging (MRI) as an alternative to CT-based treatment planning and simulation alone, especially for 
applications in adaptive ion radiotherapy. In contrast to photons, depth-dose distributions of ions are far more sensitive to whether the tissue class has been assigned correctly. This study presents therefore a reliable test if MRI-based treatment plan simulation is generally feasible.

As MRI is based on the effect of nuclear magnetic resonance, patients are not exposed to ionizing radiation during measurements. Therefore MRI provides the opportunity of patient examinations before each fraction for validation and adaptation of treatment plans. In the future, even real-time MRI-guided ion radiotherapy is imaginable, analogue to current studies with X-rays using a hybrid MRI linac modality $[2,3]$.

The major challenge in the application of MRI for treatment plan simulation is the fact that there exists no physical relationship between MR signal intensities and WEPL values. The MR signal depends mostly on the density of water and fat protons as well as tissue relaxation characteristics, whereas WEPL values are correlated to the electron density and the mean excitation energy. A two-step approach for the MRI-WEPL conversion is proposed here. First so-called pseudo CT (pCT) numbers are derived from MR signal intensities followed by a conversion to WEPL values using an empirical HLUT.

In the literature two different approaches for the derivation of $\mathrm{p} C \mathrm{~T}$ numbers from MR images can be found that are suggested for conventional photon radiotherapy treatment plan simulation [4-12] and MR/PET attenuation correction [13-17]: a first approach deals with atlas-based methods that employ non-rigid registration between a template MR image and the patient's MR image to warp a template CT image to the patient's anatomy. A second approach consists of segmentation-based methods that use a voxelwise classification of tissue into several classes that are correlated with certain bulk CT numbers.

In this work the potential of the second approach is investigated using a classifier that is based on discriminant analysis. After data preparation (Figure 1(a)), each voxel in a MR image can be associated with a certain pCT number (Figure 1(b)). The classification method was chosen as it allows a patient-specific voxelwise prediction of $\mathrm{p} \mathrm{CT}$ numbers. In comparison to atlas-based methods, it might perform better handling anatomical changes that cannot be described by a translation or deformation of voxels, e. $\mathrm{g}$. the filling of an air cavity with mucus. However, it is not the aim of this study to compare both methods.

The study consists of three parts. (1) Appropriate MR sequences and parameters are evaluated by crossvalidation studies of three tissue phantoms in order to minimize errors of $\mathrm{p} C \mathrm{CTs}$ compared to a reference CT (rCT) (Figure 1(c)). (2) The pCTs with lowest errors are used for ion radiotherapy treatment plan simulations (Figure 1(d)). For each phantom ion radiotherapy treatment plans are optimized to a planning target volume
(PTV) on both p CT and $\mathrm{r}$ CT images. The resulting fields are then both calculated on $\mathrm{r}$ CT images and compared afterwards. (3) A target movement is simulated by shifting the irradiated PTV (Figure 1(e)). Ion radiotherapy plans adapted to the new position of the PTV are optimized on a p CT. The results are compared to optimizations on a r CT without taking account of the target shift.

\section{Material and methods}

\section{Multimodal phantoms}

As specific MR sequences were employed for the present study, which are not applied at clinical radiotherapy examinations, a retrospective evaluation of patient data was not possible. Therefore three pieces of pork meat were used as multimodal phantoms. These samples were composed of fatty tissue, muscle and bones to cover a large range of MR relaxation parameters and attenuation properties for photons and ions in living tissue (Table 1).

\section{CT image acquisition}

CT image acquisition was performed on a Siemens Somatom Definition Flash CT scanner. The X-ray spectra voltage was $140 \mathrm{kVp}$ with additional tin filtration and an effective tube current-time product of $81 \mathrm{mAs}$. Images were reconstructed from raw data with a D30s kernel. The slice thickness was $1.0 \mathrm{~mm}$ and a field of view of $256 \mathrm{~mm}$ was chosen resulting in an in-plane resolution of $0.5 \mathrm{~mm}$.

\section{MR image acquisition}

MR images were acquired on a Siemens Magnetom Trio Tim 3T MRI scanner. For all samples a set of eight image series with various contrasts was created. The imaging of bones using standard MR sequences leads to very low signal intensities similar to air due to the very short T2 relaxation constant of bony tissue. Therefore a 3D ultrashort echotime (UTE) sequence with radial k-space sampling was employed, that allows for distinguishing bone tissue from air [19,22-24]. In addition, a 2D turbo spin echo (TSE) sequence with proton density- and T2-weighting and a 3D ultrafast gradient echo (MPRAGE) sequence with magnetization preparation for T1-weighting were used (Table 2). All sequences had the same field of view of $256 \mathrm{~mm}$. The TSE and MPRAGE sequences had a nominal resolution of $1.0 \times 1.0 \times 2.0 \mathrm{~mm}^{3}$, whereas the nominal resolution of the UTE sequence was isotropic with $1.0 \times 1.0 \times 1.0 \mathrm{~mm}^{3}$. An automatic distortion correction filter and a correction of inhomogeneous coil illumination was activated in each sequence protocol.

\section{Image registration and masking}

All acquired MR images were coregistered to their corresponding CT images with a rigid registration algorithm based on maximization of mutual information [25]. MR images were resampled to $\mathrm{CT}$ resolution in all three 


\section{(a) data preparation}

learning step:

MR images (up to 8 different contrasts)
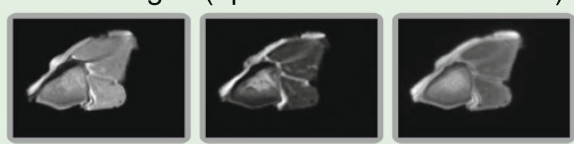

CT image

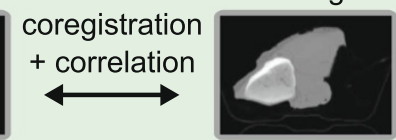

+ additional features

prediction step:

MR images (up to 8 different contrasts)
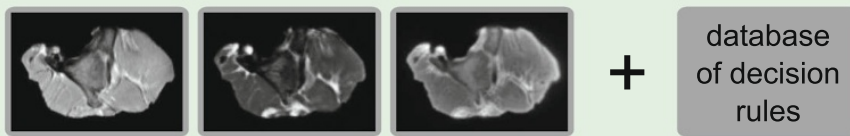

+ additional features

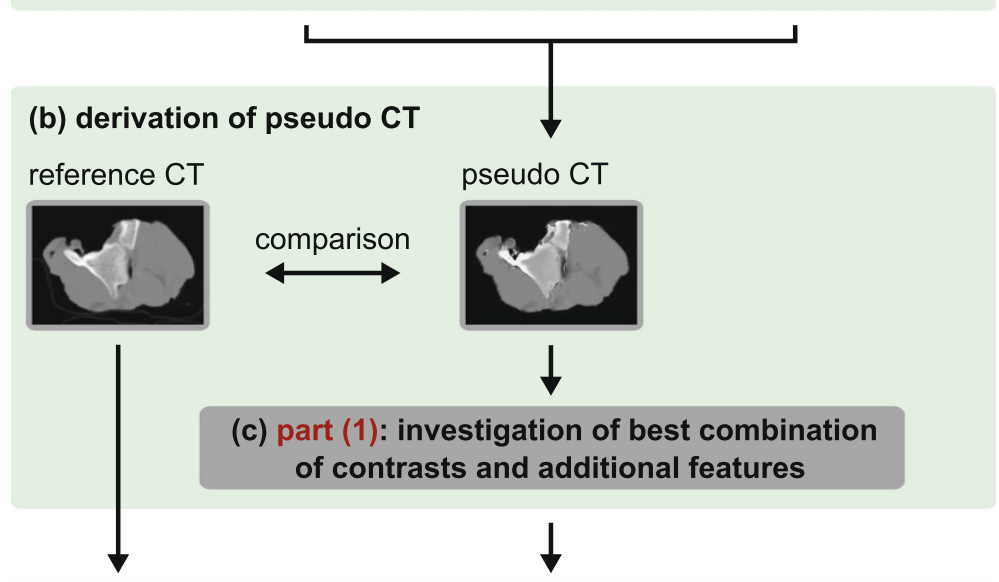

(d) part (2): ion radiotherapy treatment plan simulation

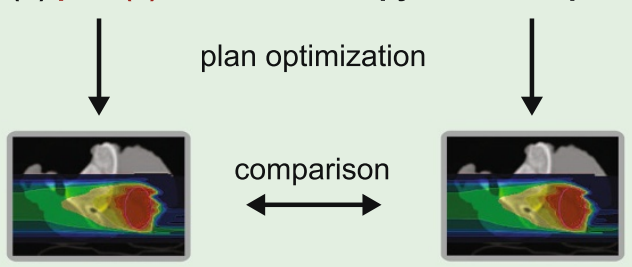

(e) part (3): ion radiotherapy treatment plan adaptation

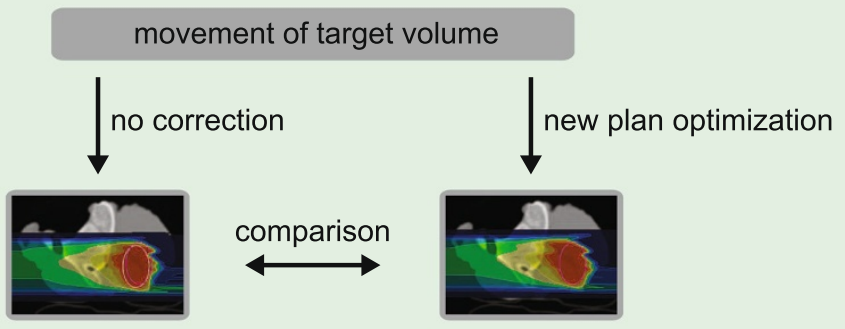

Figure 1 Experiments and workflow of MRI-based ion radiotherapy treatment plan simulation and adaptation. (a) data preparation for classification; (b) derivation of pCT numbers from MR images; (c) cross-validation study for investigating the best combination of contrasts and additional features; (d) ion radiotherapy treatment plan simulation; (e) ion radiotherapy treatment plan adaptation. 
Table 1 MR- and CT-related parameters for tissue from literature

\begin{tabular}{lrrr}
\hline Tissue & $\begin{array}{r}\text { Relative electron } \\
\text { density } \rho_{\mathbf{e}}\end{array}$ & T1 [ms] & T2 [ms] \\
\hline $\begin{array}{l}0.951^{a} \\
\text { subcutaneous fat }\end{array}$ & $0.982^{a}$ & $371^{d}$ & $133^{d}$ \\
$\begin{array}{l}\text { skeleton - yellow } \\
\text { marrow }\end{array}$ & $1.035^{a}$ & $1084^{c}$ & $133^{d}$ \\
$\begin{array}{l}\text { brain - white } \\
\text { matter }\end{array}$ & $1.035^{a}$ & $1820^{c}$ & $69^{c}$ \\
$\begin{array}{l}\text { brain - gray } \\
\text { matter }\end{array}$ & $1.040^{a}$ & $1412^{c}-1420^{d}$ & $32^{d}-50^{c}$ \\
$\begin{array}{l}\text { muscle } \\
\text { kidney }\end{array}$ & $1.041^{a}$ & $1194^{c}$ & $56^{c}$ \\
$\begin{array}{l}\text { blood } \\
\text { liver }\end{array}$ & $1.050^{a}$ & $1932^{c}$ & $275^{c}$ \\
$\begin{array}{l}\text { skeleton - } \\
\text { cartilage } \\
\text { skeleton - } \\
\text { cortical bone }\end{array}$ & $1.050^{a}$ & $812^{c}$ & $42^{c}$ \\
\hline
\end{tabular}

${ }^{a}[18], b[19],{ }^{c}[20],{ }^{d}[21]$. All measurements were performed in vivo at $3 T$, values for cortical bone were measured at $1.5 \mathrm{~T}$ and represent the relaxation time T2*

dimensions by linear interpolation to make a voxelwise correlation of images possible. Afterwards, voxels at the image edges consisting of air were removed by a threshold mask in order to reduce computing time for additional feature extraction and classification. The following calculations refer only to voxels within that mask.

\section{Additional feature extraction}

Additional features were extracted for each voxel from its 26 surrounding voxels of a $3 \times 3 \times 3$ box according to [10] and its location in order to augment the information provided by the signal intensities of voxels:

- box.mean: the mean intensity of the surrounding box including the central voxel
- box.sd: the standard deviation of the surrounding box including the central voxel multiplied by the intensity of the voxel

- dist.xyz: the three absolute distances of the voxel to the center of the 3D imaging volume in each spatial dimension

- dist.center: the absolute distance of the voxel to the center of the 2D slice

Neighborhood-related parameters were employed for improving results at transitions, e.g. standard deviations of boxes at transitions were larger than at homogeneous sites. Extracting coodinate-related features assumed a certain symmetric spatial distribution of tissue classes, in particular a cylindrical symmetry for dist.center and a spherical symmetry for dist.xyz. Before calculating distances of voxels to the image center, coordinates of voxels were translated so that samples were centered in the coordinate system of images.

\section{Discriminant analysis}

The idea of discriminant analysis is to assign one of $k$ predefined classes $C_{1}, C_{2}, \ldots, C_{k}$ to an observation variables vector $\mathbf{X}=\left(x_{1}, x_{2}, \ldots, x_{p}\right)^{\mathrm{T}} \in \mathbb{R}^{p}$ with unknown class membership [26]. In the learning step a learning data set that consists of $n$ observation variables vectors $\mathbf{X}_{i}$ labeled with their corresponding classes $C_{i}$ is employed to derive a decision rule that can associate a new observation variables vector $\mathbf{X}$ with one of the $k$ classes (Figure 1(a)). Class memberships of new observation variable vectors can then be predicted using the decision rule (Figure 1(b)).

Here, each observation variables vector $\mathbf{X}_{j}$ was composed of intensities of several MRI contrasts and additionally extracted features of one particular voxel $j$ of the coregistered MR images. Class membership of a voxel was obtained from its CT number. Therefore the CT scale was divided into 128 sections each having a range of $32 \mathrm{HU}$ and each voxel $j$ was assigned with its appropriate CT class $C_{j}$.

Table 2 Parameter settings of MR sequences

\begin{tabular}{|c|c|c|c|c|c|}
\hline Sequence & $\begin{array}{r}\text { Echo time } \\
{[\mathrm{ms}]}\end{array}$ & $\begin{array}{r}\text { Repetition time } \\
{[\mathrm{ms}]}\end{array}$ & $\begin{array}{r}\text { Flip angle } \\
{\left[{ }^{\circ}\right]}\end{array}$ & $\begin{array}{r}\text { Scan time } \\
\text { [min] }\end{array}$ & Additional parameters \\
\hline TSE1 & 8.3 & 7500 & 90 & 4.8 & \\
\hline TSE2 & 8.3 & 7500 & 90 & 4.8 & with fat saturation \\
\hline TSE3 & 75.0 & 7500 & 90 & 3.8 & \\
\hline MPRAGE & 2.38 & 2000 & 12 & 6.4 & inversion time $=700 \mathrm{~ms}$ \\
\hline \multirow[t]{2}{*}{ UTE1 } & 0.05 & 7.25 & 12 & 6.1 & radial views $=50001$ \\
\hline & 4.91 & & & & \\
\hline \multirow[t]{2}{*}{ UTE2 } & 0.05 & 7.25 & 60 & 6.1 & radial views $=50001$ \\
\hline & 4.91 & & & & \\
\hline
\end{tabular}




\section{Classification algorithm}

For classification of voxels into CT classes the high dimensional discriminant analysis (HDDA) algorithm from the package "HDclassif" (version 1.2.1 [26,27]) implemented in $\mathrm{R}$ (The R Foundation for Statistical Computing, version 2.14.2 [28]) was chosen as a reliable, well-tested and efficient method. It is closely related to the well-known quadratic discriminant analysis (QDA). According to the classical Gaussian mixture model framework, HDDA assumes that class conditional densities are Gaussian. Thus, a certain variation of absolute MR signal intensities as observation variables, e.g. due to inhomogeneous coil illumination, was taken into account. To increase efficiency in computing time compared to QDA, the assumption is made that high-dimensional data live around subspaces with a lower dimension than $p$. Therefore the number of dimensions is reduced during the learning step and for each of the $k$ classes only the dimensions which contain most information for discrimination from other classes are maintained. In the HDDA parameter settings the most general model $a_{k j} b_{k} Q_{k} d_{k}$ was used with a threshold for dimension selection of 0.2. In addition the datasets were scaled so that each observation variable was equally weighted having a mean value of 0 and a standard deviation of 1 . The $\mathrm{p} C \mathrm{~T}$ number of a voxel was then obtained by the dot product of the posterior probabilities of a voxel for each of the $k$ classes and the mean $\mathrm{CT}$ number of each class calculated from the learning dataset.

\section{Ion radiotherapy treatment plan simulation}

Ion radiotherapy plans based on $\mathrm{r} C \mathrm{C}$ or $\mathrm{p} C \mathrm{C}$ images were created using the treatment planning system VIRTUOS developed at DKFZ [29]. Prescribed physical doses were 3 Gy for protons and 1 Gy for carbon ions. Irradiation fields were optimized and simulated with the software TRiP98 from GSI, Darmstadt (Germany) [30,31]. A rasterscanning pencil beam for protons and carbon ions with a FWHM of $5 \mathrm{~mm}$ passing a $3 \mathrm{~mm}$ ripple filter was assumed. WEPL values were obtained from $\mathrm{rCT}$ or $\mathrm{pCT}$ images with an empirical HLUT that was derived from $140 \mathrm{kVp}$ $\mathrm{CT}$ measurements of Gammex tissue equivalent materials (Figure 2).

\section{Ion radiotherapy treatment plan adaptation}

In order to investigate the potential of MRI for treatment plan adaptation, i.e. to align plans with interfractional anatomical changes, a target volume movement was simulated by shifting the irradiated PTV of meat sample 2 . Then, optimizations of carbon ion and proton treatment plans created in VIRTUOS and adapted to the target shift were performed on p CT images with TRiP98 and compared to a r CT optimization without plan adaptation.

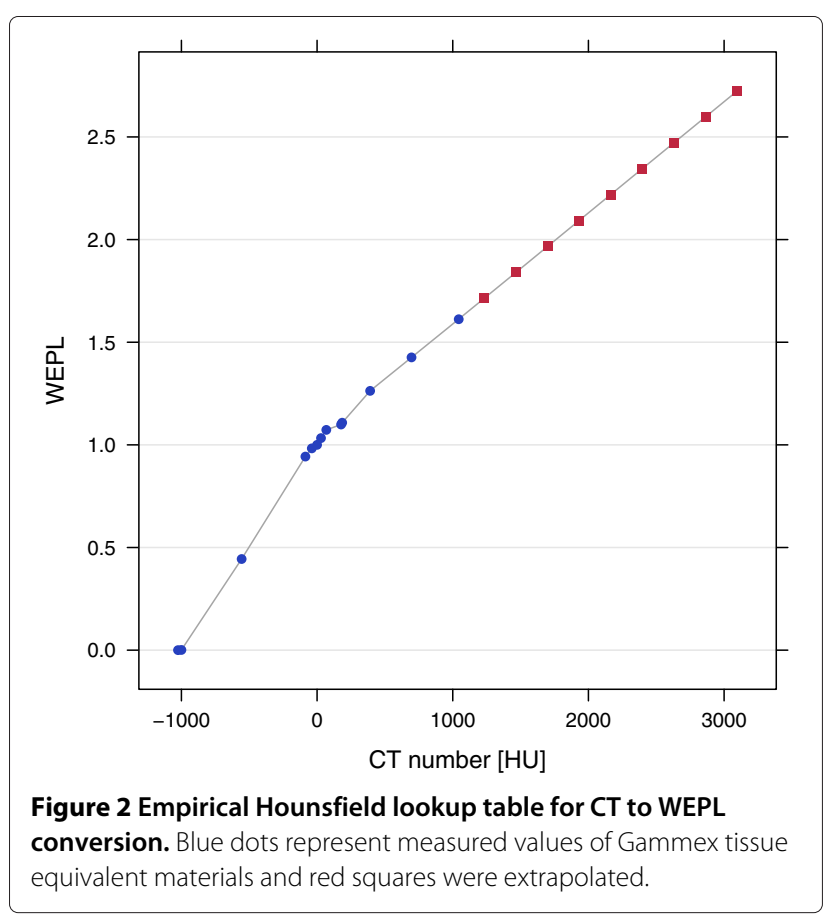

\section{Results}

Derivation of pseudo CT numbers from MR images

For all three samples, CT images and MR images with eight different contrasts were acquired and coregistered. In a cross-validation study, for each sample a p CT was predicted from MR images using the other two samples for creating the learning database. 738 different combinations of contrasts and additional features as input of the observation variables vector $\mathbf{X}$ were tested for datasets

\section{Table 3 Results of cross-validation study}

\begin{tabular}{llr}
\hline Contrasts & Additional features & $\begin{array}{r}\text { Mean absolute } \\
\text { error [HU] }\end{array}$ \\
\hline TSE1, UTE2 & box.sd, dist.center & 92.5 \\
TSE1, UTE2 & box.sd, dist.xyz & 93.5 \\
TSE1, UTE1 & box.sd, dist.center & 94.1 \\
TSE1, UTE2 & box.sd, dist.center, dist.xyz & 95.8 \\
TSE1, UTE2 & box.mean, box.sd, dist.center & 96.5 \\
TSE1, UTE2 & box.mean, box.sd, dist.xyz & 96.5 \\
TSE1, MPRAGE, UTE2 & box.sd, dist.center & 96.7 \\
TSE1, TSE2, UTE2 & box.sd, dist.center & 97.0 \\
\hline TSE1, UTE1, UTE2 & none & 111.7 \\
UTE1 & box.mean, box.sd, dist.xyz & 100.7 \\
TSE1, MPRAGE & box.mean, box.sd, dist.center & 115.7 \\
\hline
\end{tabular}

Mean absolute errors of masked voxels for the 8 best combinations of contrasts and additional features out of 738 combinations in comparison to the best result without any additional feature as well as without TSE1 and UTE sequences.

Results were obtained for reduced datasets of 32 images per sample. 


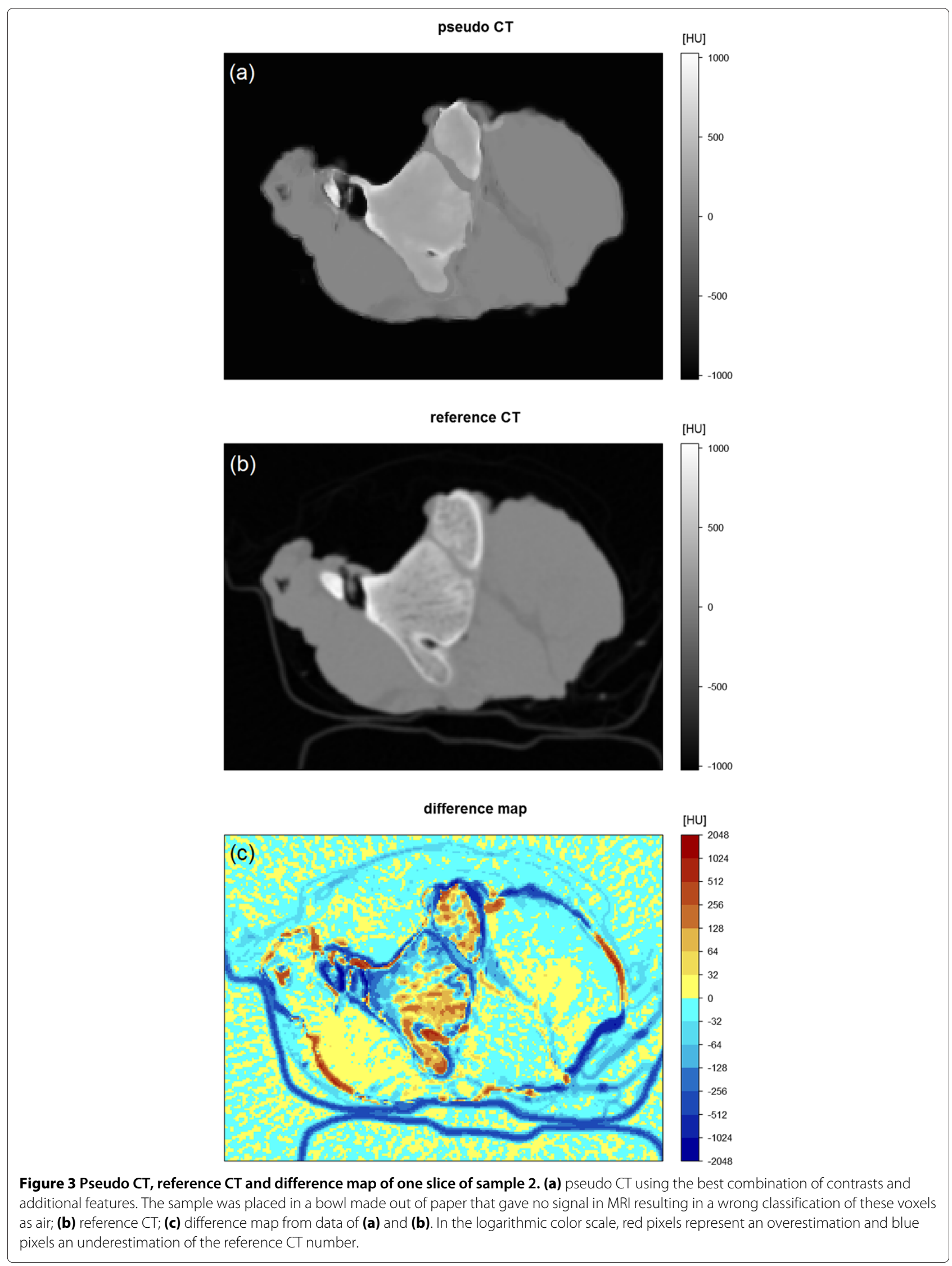




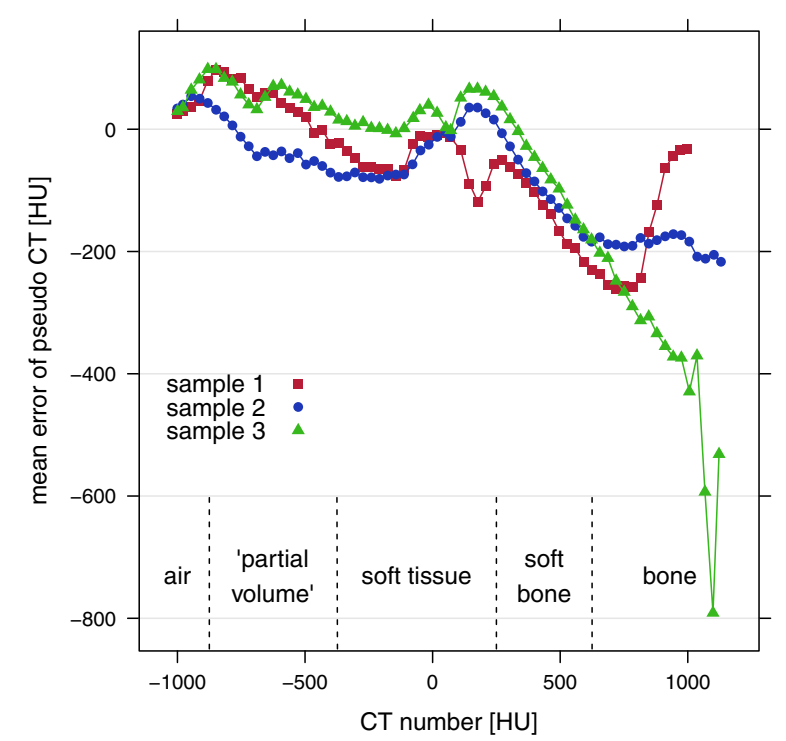

Figure 4 Mean errors of $\mathrm{p} \mathrm{CT}$ per class for the three samples. The best combination of contrasts and additional features was used for pCT prediction.

with a reduced number of 32 images per sample. For all these combinations, the mean absolute errors MAE for masked voxels were calculated by a voxelwise comparison of p CT and r CT [10]:

$$
\mathrm{MAE}=\frac{1}{n} \sum_{i=1}^{n}\left|\mathrm{pCT}_{i}-\mathrm{rCT}_{i}\right|
$$

The best results were achieved with a combination of the proton density weighted TSE sequence (TSE1) and the UTE 2 sequence each with additional features box.sd as well as dist.center resulting in a 7 dimensional observation variables vector $\mathbf{X}$ (Table 3 ). The mean absolute errors for such a combination for the full datasets were $\mathrm{MAE}_{1}=81.0 \mathrm{HU}, \mathrm{MAE}_{2}=95.2 \mathrm{HU}$ and $\mathrm{MAE}_{3}=90.1$ $\mathrm{HU}$ for samples 1-3, respectively. Variations of absolute errors between voxels were large leading to standard deviations of absolute errors between $130 \mathrm{HU}$ and $152 \mathrm{HU}$. The above described combination needed around 5-6 min of computing time for both the learning step and the prediction step (running on an Intel Core i5 processor with 4 cores for sets of 200 images for learning and 100 images for prediction). Each image was represented by a matrix of 43560 voxels. For further analysis, the $\mathrm{r} C \mathrm{~T}$ (Figure 3(b)) was subtracted from the p CT for each sample using the best combination of contrasts (TSE1, UTE2) and additional features (box.sd, dist.center) (Figure 3(a)) yielding a difference map (Figure 3(c)).

In addition the mean errors of $\mathrm{p} C \mathrm{~T}$ per class $\mathrm{ME}_{c l}$ for all three samples were calculated:

$$
\mathrm{ME}_{c l}=\frac{1}{n_{c l}} \sum_{i=1}^{n_{c l}} \mathrm{pCT}_{i, c l}-\mathrm{rCT}_{i, c l} .
$$

The dependence of MEs on CT numbers was similar for all three samples and showed a strong systematical underestimation for bony tissue (Figure 4).

\section{Ion radiotherapy treatment plan simulation}

Pseudo CT numbers and corresponding errors of the three samples were translated into pseudo WEPL values and errors by means of the $140 \mathrm{kVp}$ empirical HLUT (Figure 2). From this an estimate of the mean deviation of water equivalent thickness (WET) for five tissue classes, namely air, "partial volume", soft tissue, soft bone and bone was calculated:

$$
\Delta \mathrm{WET}_{\text {tissue }}=\Delta \mathrm{WEPL}_{\text {tissue }} \times d,
$$

with $d$ as tissue thickness. For evaluation of variations of $\triangle$ WET the standard error per tissue for a beam passing three different thicknesses was computed assuming an irradiation in a lateral direction where the image resolution was $0.5 \mathrm{~mm}$. Similar to the calculation of mean errors of $\mathrm{p}$ CT per class, very dense bone classes were significantly underestimated, whereas the air and the "partial volume" class exhibited an overestimation. Soft tissue was in good agreement with reference values (Figure 4 and Table 4).

For treatment plan simulations, PTVs were placed in each sample at a position considered to be the most challenging, i.e. the ion beam had to pass bony structures.

\begin{tabular}{|c|c|c|c|c|}
\hline Tissue & $\begin{array}{r}\text { Mean error of } \\
\text { p CT [HU] }\end{array}$ & $\begin{array}{r}\Delta \mathrm{WET}_{1 \mathrm{~cm}} \\
{[\mathrm{~mm}]}\end{array}$ & $\begin{array}{r}\Delta \mathrm{WET}_{5 \mathrm{~cm}} \\
{[\mathrm{~mm}]}\end{array}$ & $\begin{array}{r}\Delta \mathrm{WET}_{10 \mathrm{~cm}} \\
{[\mathrm{~mm}]}\end{array}$ \\
\hline air & 43.3 & $0.4 \pm 0.3$ & $1.8 \pm 0.6$ & $3.6 \pm 0.9$ \\
\hline "partial volume" & 28.6 & $0.3 \pm 0.7$ & $1.4 \pm 1.5$ & $2.9 \pm 2.1$ \\
\hline soft tissue & -1.4 & $-0.02 \pm 0.3$ & $-0.1 \pm 0.6$ & $-0.2 \pm 0.9$ \\
\hline soft bone & -63.2 & $-0.4 \pm 0.3$ & $-1.8 \pm 0.6$ & $-3.7 \pm 0.9$ \\
\hline bone & -223.1 & $-1.2 \pm 0.4$ & $-6.0 \pm 0.8$ & $-11.9 \pm 1.1$ \\
\hline
\end{tabular}
After $\mathrm{pCT}$ and $\mathrm{rCT}$ conversion to WEPL values, proton

Table 4 Mean errors of p CT and estimated mean deviations of WET for different tissue types

The best combination of contrasts and additional features was used for calculations. 

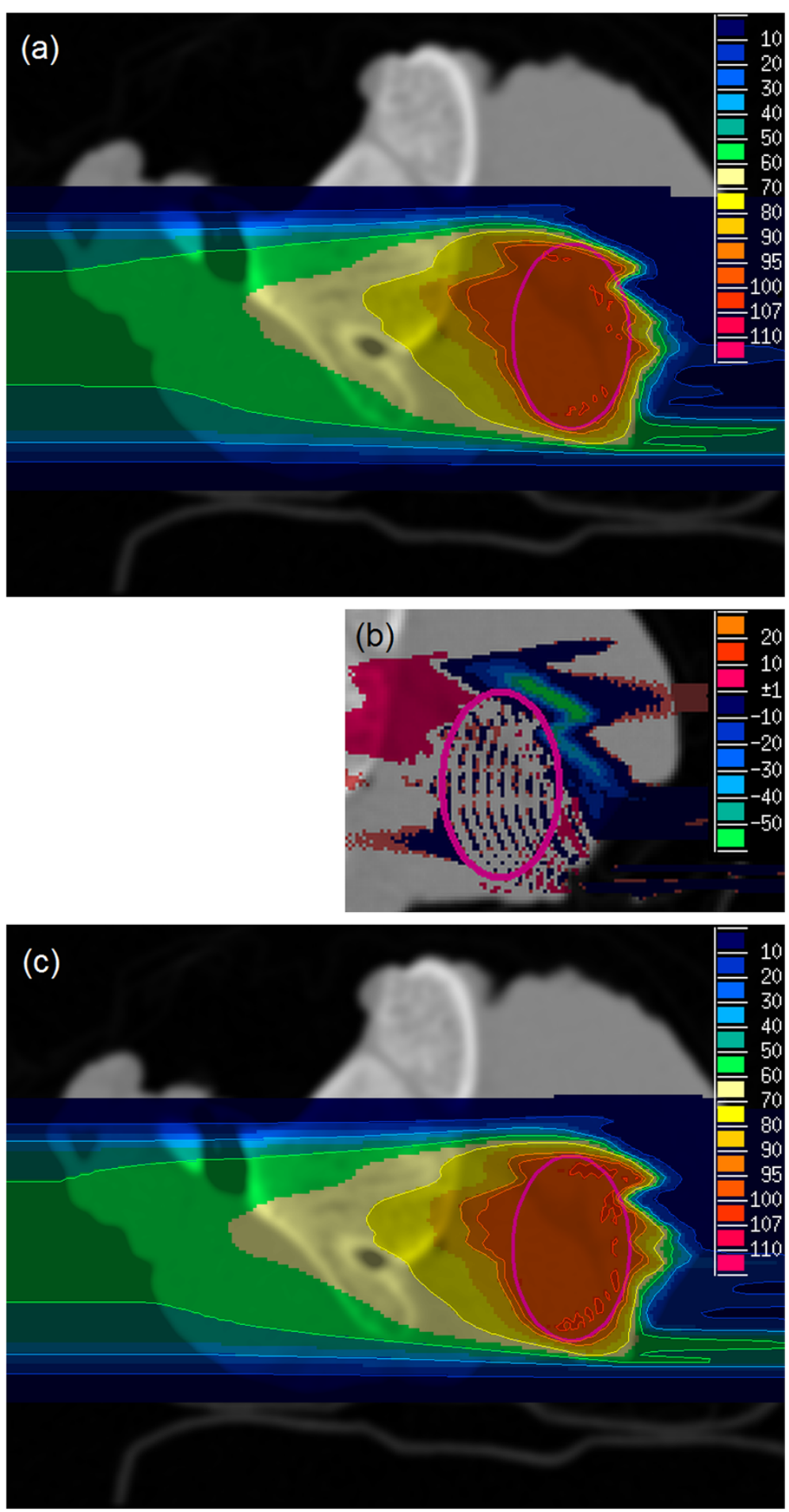

Figure 5 Carbon ion plans of sample 2. (a) optimized on $\mathrm{pCT}$ and recalculated on $\mathrm{rCT}$; (b) dose difference of (a) and (c); (c) optimized and calculated on $r$ CT. The PTV size was $17 \times 26 \times 16 \mathrm{~mm}^{3}$ and the unit of color scale is percent of the prescribed physical dose. 
and carbon field optimizations were run on both MRIbased pCT images as well as on $\mathrm{rCT}$ images for all three samples. The fields optimized on MRI-based pCTs were recalculated on $\mathrm{rCTs}$ that were considered to be the gold standard (Figure 5(a)). The results were compared with plans optimized and calculated on $\mathrm{r} C \mathrm{~T}$ images (Figure 5(b), (c)).

Dose-Volume histograms (DVH) were computed for the fields optimized on MRI-based p CTs and recalculated on r CTs (Figure 6 red curve) as well as for fields optimized and calculated on $\mathrm{rCTs}$ (Figure 6 blue curve). Table 5 shows the corresponding dose statistics parameters mean dose of PTV and volume of PTV with dose less than $90 \%$ of prescribed dose.

\section{Ion radiotherapy treatment plan adaptation}

The irradiated PTV of sample 2 was shifted $2.0 \mathrm{~mm}$ in $x$ and $2.0 \mathrm{~mm}$ in $y$-direction for simulating a target volume movement (Figure 7(b)).

Proton and carbon field optimizations were run on the pCT using a plan adapted to the shifted PTV. The optimized fields were recalculated on the $\mathrm{r} C \mathrm{~T}$ with the shifted PTV (Figure 7(a)). The results were compared to fields optimized on $\mathrm{rCT}$ before PTV shift and recalculated on the $\mathrm{r}$ CT after PTV shift without plan adaptation (Figure 7(b)). DVHs were computed for both optimizations (Figure 8). Adapted pCT optimizations showed slightly better results than non-adapted $\mathrm{r} C \mathrm{~T}$ optimizations having comparable mean doses of PTV and 2.0 $2.4 \%$ smaller volumes of PTV with doses less than $90 \%$ of prescribed dose.

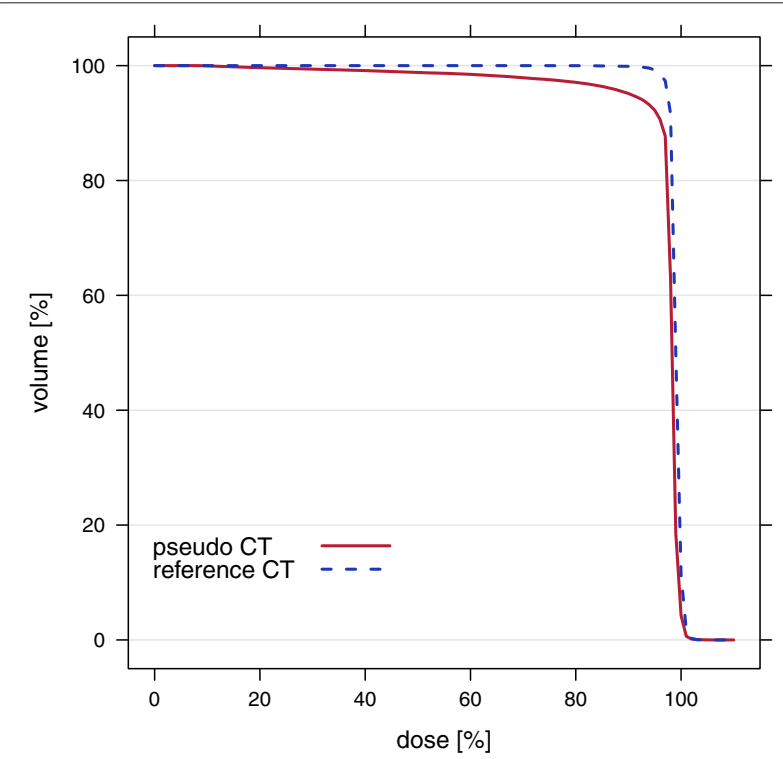

Figure 6 Dose-volume histograms of sample 2 for carbon plans. Optimized on MRI-based p CT and recalculated on $\mathrm{rCT}$ (red curve) as well as optimized and calculated on rCT (blue curve).

\section{Discussion}

\section{Derivation of pseudo CT numbers from MR images}

The cross-validation study with different combinations of contrasts and additional features revealed remarkable results: (1) the UTE sequence was essential for the distinction of bony tissue and air and could reduce MAE by $20 \%$ (Table 3). (2) Proton density weighted images seemed to contain the most information for the derivation of $\mathrm{pCT}$ values compared to all other tested contrasts besides the UTE sequence. (3) Additional feature extraction could reduce the MAE by $17 \%$. In general, p CT numbers of water-like soft tissue around $0 \mathrm{HU}$ had the lowest absolute errors compared to the $\mathrm{r}$ CT (Figure 4). However, MEs could not be smaller than $\pm 16 \mathrm{HU}$ in average as the CT scale was divided into sections of $32 \mathrm{HU}$.

Compact bone classes showed a strong systematical underestimation. One reason for this comes from an imperfect signal discrimination of bones and other tissue types using the UTE sequence. In particular fattytissue showed a strong signal at UTE images that were acquired at very short echo times. Another cause for the underestimation is related to clipping errors. Bone tissue classes represented the classes with highest CT numbers. Therefore either a correct classification or an underestimation was possible producing too low $\mathrm{CT}$ numbers on average.

Largest errors could be found at transitions from air to soft tissue, soft tissue to bone and air to bone. These intermediate regions between different tissue classes included single voxels with deviations of more than $1500 \mathrm{HU}$. In particular partial volume effects, errors of registration and image resampling by linear interpolation were responsible for these large errors leading to a wrong classification on the CT scale.

In the extraction of additional features, the box.sd feature contributed most to the improvements (Table 3). Near borders the standard deviation of a box surrounding a voxel was higher than in homogeneous tissue. This additional information used as a further dimension of the observation variables vector improved discrimination of "partial volume" and other tissue classes and spots at borders with deviations larger than $500 \mathrm{HU}$ could be reduced significantly. The coordinate-related features dist.xyz and dist.center both also decreased the MAE, showing that the spatial distribution of several tissue classes had certain degrees of cylindrical and spherical symmetries.

In the following, several opportunities are proposed for improving the classification results:

- an extended set of samples (more than two) should be employed for creating the learning database of decision rules to compensate for anomalies and differences between samples 
Table 5 Dose statistics parameters of carbon ion and proton treatment plans

\begin{tabular}{|c|c|c|c|c|c|}
\hline \multirow{3}{*}{ Sample type } & \multirow{3}{*}{ Beam modality } & \multicolumn{2}{|c|}{ p CT optimization } & \multicolumn{2}{|c|}{ r CT optimization } \\
\hline & & Mean & Volume with dose & Mean & Volume with dose \\
\hline & & dose [\%] & $<90 \%[\%]$ & dose [\%] & $<90 \%[\%]$ \\
\hline \multirow[t]{2}{*}{ meat sample 1} & carbon & 96.5 & 8.4 & 99.0 & 0.7 \\
\hline & proton & 96.2 & 8.3 & 99.3 & 0.0 \\
\hline \multirow[t]{2}{*}{ meat sample 2} & carbon & 96.7 & 4.6 & 99.0 & 0.1 \\
\hline & proton & 96.2 & 6.0 & 98.9 & 0.4 \\
\hline \multirow[t]{2}{*}{ meat sample 3} & carbon & 97.4 & 3.4 & 98.8 & 0.3 \\
\hline & proton & 97.3 & 2.4 & 98.8 & 0.2 \\
\hline
\end{tabular}

Plans were optimized on $\mathrm{pCT}$ as well as $\mathrm{rCT}$ and both optimizations were recalculated on $\mathrm{rCT}$ for comparison of parameters.
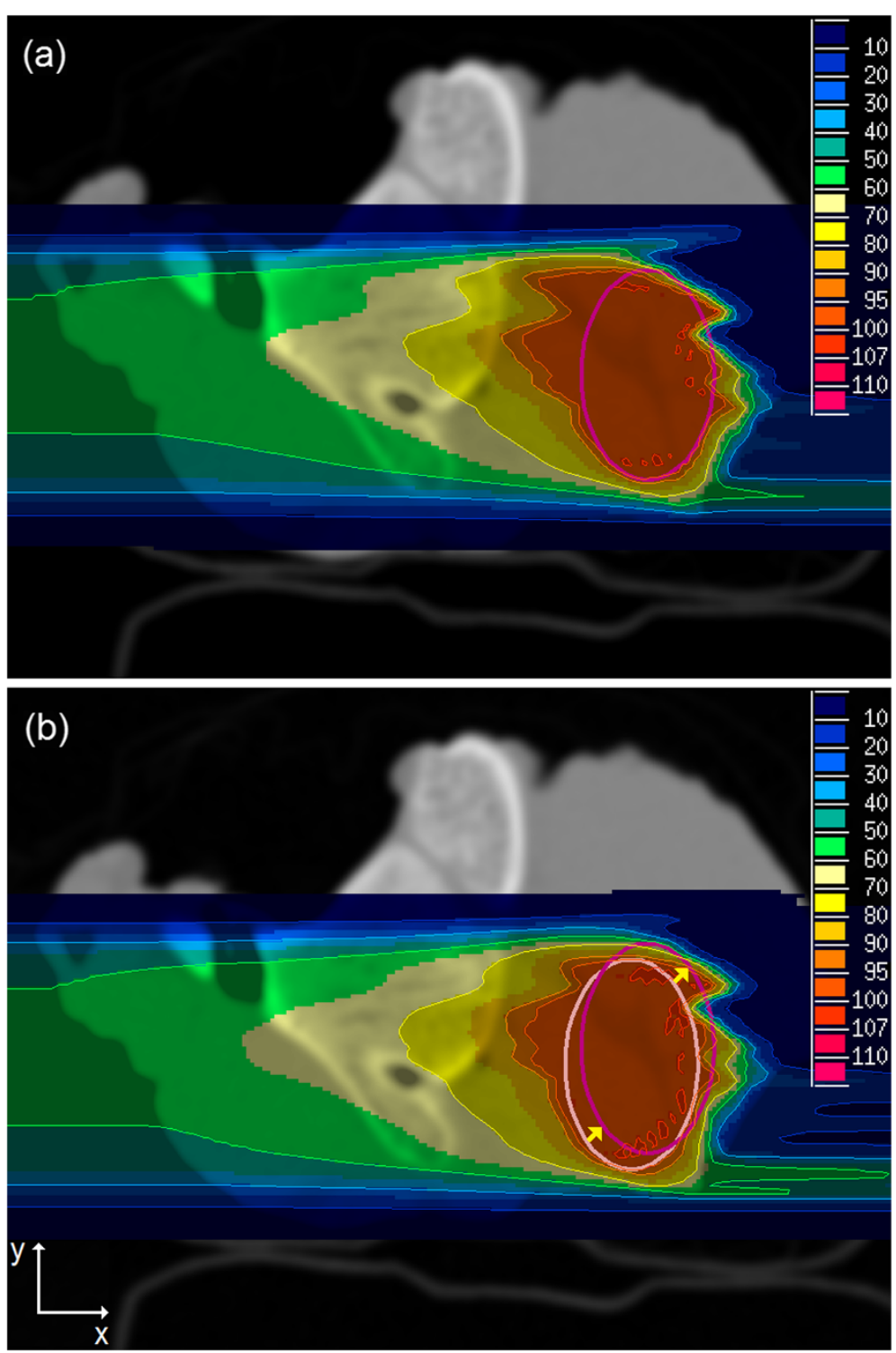

Figure 7 Carbon ion plans of sample 2 with shifted PTV. The old PTV (light magenta) was shifted $2.0 \mathrm{~mm}$ in $x$ - and $2.0 \mathrm{~mm}$ in $y$-direction to a new position (dark magenta): (a) adapted plan optimized on $\mathrm{PCT}$ after PTV shift and recalculated on $\mathrm{rCT}$, (b) optimized on $\mathrm{rCT}$ before PTV shift and recalculated on $\mathrm{rCT}$ after PTV shift without plan adaptation. The unit of color scale is percent of the prescribed physical dose. 


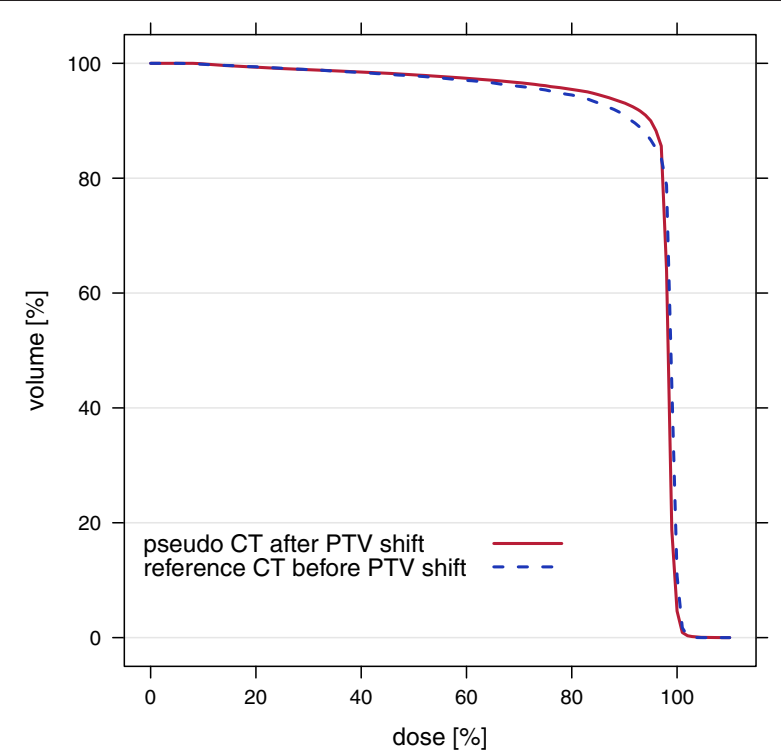

Figure 8 Dose-volume histograms of sample 2 for carbon plans with shifted PTV. Optimized on p CT after PTV shift (red curve) and on $r$ CT before PTV shift without plan adaptation (blue curve). Both plans were recalculated on the $\mathrm{rCT}$ after PTV shift.

- more suitable MRI contrast parameters may have to be found for a further improved discrimination of tissue classes

- an UTE sequence with fat saturation might improve the distinction of bones and other tissue, in particular fatty tissue

- a better correction of inhomogeneous coil illumination than the implemented filter might decrease the variances of MR signal intensities of the different classes

- further additional features, e. g. larger box sizes of $7 \times 7 \times 7$ voxels for mean and standard deviation might reduce the effects of partial volume and interpolation leading to smaller errors at tissue transitions

- CT scale may be divided into non-equidistant sections or other ways of class determination may exist, e. g. dependent on spatial location of a voxel combined with its CT number

- more sophisticated discrimination tools like nonparametric discriminant analysis or support vector machines may have to be used instead of HDDA

\section{Ion radiotherapy treatment plan simulation}

In the simulation of ion radiotherapy treatment plans, MRI-based pCT optimized plans had small deviations compared to $\mathrm{rCT}$ optimized plans that were assumed to be the gold standard. Dose coverage of PTVs was slightly better for $\mathrm{rCT}$ optimizations than for $\mathrm{pCT}$ optimizations (Figure 5 and Table 5). In particular, mean doses of
PTVs were 1.4 - 3.1\% higher, whereas volumes of PTVs with doses less than $90 \%$ of prescribed dose were 2.2 8.3\% smaller. Especially distal regions of PTVs showed an underdosage in p CT optimizations. This was expected as the ions had to pass bony structures that were systematically underestimated in p CT images.

The DVHs also revealed a better dose coverage of PTVs for $\mathrm{rCT}$ optimizations compared to $\mathrm{p} \mathrm{CT}$ optimizations (Figure 6). These results demonstrated that the classification method for deriving $\mathrm{p} C \mathrm{C}$ numbers from MR images performed well in the soft tissue region, whereas for dense materials like compact bones errors were large. However, the effect of these large errors on dose dostributions of treatment plan simulations using $\mathrm{pCT}$ for optimization was relatively small. This can be explained by the composition of bones having only a thin outer layer of compact bone. Therefore the proportion of voxels with these large errors was small.

\section{Ion radiotherapy treatment plan adaptation}

MRI-based treatment plan adaptation was tested by simulating a movement of target volume. A plan optimized on pCT and adapted to the PTV shift was compared to a plan optimized on $\mathrm{r}$ CT before PTV shift. The adapted plan optimized on p CT showed a slightly better dose coverage of the shifted target volume as the $\mathrm{r} C \mathrm{~T}$ plan without plan adaptation (Figures 7, 8).

This result indicated that the error introduced by using a pCT for optimization was comparable to the error resulting from a target volume shift of $2.0 \mathrm{~mm}$ in $x$ - and $y$ direction. In this particular case, MRI examinations before each fraction and a treatment plan adaptation to interfractional changes of anatomy or tumor movements would improve dose coverage of PTV for target volume shifts larger than $2.0 \mathrm{~mm}$ in two directions. In order to reduce errors using $\mathrm{p} \mathrm{CT}$, a combination of the original $\mathrm{r} C \mathrm{C}$ with a p CT might be employed for adaptation of treatment plans. As the bone tissue class had largest deviations in pCT images and anatomical changes in bony structures are uncommon, solely soft tissue regions of the $\mathrm{rCT}$ in which anatomical changes are found in MRI could be substituted by pCT values. However, this approach might also introduce registration errors and a careful evaluation of advantages and disadvantages is necessary.

\section{Conclusions}

In this study the potential of MRI for treatment plan simulation and adaptation in ion radiotherapy was investigated. It was shown that a MRI-based derivation of pCT values using a statistical classification approach is feasible although no physical relationship between MR signal intensities and X-ray CT numbers exists. Results obtained in cross-validation studies of three tissue samples showed a strong underestimation of compact bone 
classes resulting from an imperfect distinction of bones and other tissue types applying the UTE MR sequence. In simulations of treatment plans these deviations revealed mean doses of PTVs being $1.4-3.1 \%$ smaller for p CT optimizations compared to $\mathrm{r} C \mathrm{~T}$. Considering adaptation of treatment plans, these deviations corresponded to uncertainties introduced by an interfractional target volume shift of $2 \mathrm{~mm}$ in two directions.

In the future MRI may complement the treatment planning process for ion radiotherapy and improve the accuracy so as to reap the rewards of highly conformal irradiation of tumors with charged particles. Especially applications for adaptive ion radiotherapy seem to be interesting as MRI examinations before each fraction would give the opportunity of adaptation to interfractional changes of anatomy without additional dose to the patient.

\section{Competing interests}

The authors declare that they have no competing interests.

\section{Authors' contributions}

CMR designed the study, carried out the MRI and CT measurements, developed the framework for data evaluation, performed the statistical analysis and drafted the manuscript. CT measured data for the HLUT for CT to WEPL conversion. NH supported the CT measurements and helped to draft the manuscript. AMN supported the MRI measurements and helped to draft the manuscript. OJ participated in the design of the study and helped to draft the manuscript. SG initiated the study, participated in its design and coordination and helped to draft the manuscript. All authors read and approved the final manuscript.

\section{Acknowledgements}

We would like to thank Andreas Jäger (German Cancer Research Center) for his support with multimodal image registration.

\section{Author details \\ ${ }^{1}$ German Cancer Research Center (DKFZ), Division of Medical Physics in Radiation Oncology, Im Neuenheimer Feld 280, 69120 Heidelberg, Germany. ${ }^{2}$ German Cancer Research Center (DKFZ), Division of Medical Physics in Radiology, Im Neuenheimer Feld 280, 69120 Heidelberg, Germany. ${ }^{3}$ Heidelberg University Hospital, Department of Radiation Oncology, Im Neuenheimer Feld 400, 69120 Heidelberg, Germany.}

Received: 12 November 2012 Accepted: 11 February 2013 Published: 6 March 2013

\section{References}

1. Schardt D, Elsässer T, Schulz-Ertner D: Heavy-ion tumor therapy: physical and radiobiological benefits. Rev Mod Phys 2010, 82:383-425. [http://link.aps.org/doi/10.1103/RevModPhys.82.383].

2. Lagendijk JJW, Raaymakers BW, Raaijmakers AJE, Overweg J, Brown KJ, Kerkhof EM, van der PutRW, Hå rdemark B, van Vulpen M, van der Heide UA: MRI/linac integration. Radiother Oncol 2008, 86:25-29. [http://www.ncbi.nlm.nih.gov/pubmed/18023488].

3. Fallone BG, Murray B, Rathee S, Stanescu T, Steciw S, Vidakovic S, Blosser E, Tymofichuk $D$ : First MR images obtained during megavoltage photon irradiation from a prototype integrated linac-MR system. Med Phys 2009, 36(6):2084-2088. [http://ink.aip.org/link/MPHYA6/v36/i6/ p2084/s1\&Agg=doi].

4. Lee YK, Bollet M, Charles-Edwards G, Flower MA, Leach MO, McNair H, Moore E, Rowbottom C, Webb S: Radiotherapy treatment planning of prostate cancer using magnetic resonance imaging alone. Radiother
Oncol 2003, 66(2):203-216. [http://www.ncbi.nlm.nih.gov/entrez/query. fcgi?cmd=Retrieve\&db=PubMed\&dopt=Citation\&list_uids=12648793].

5. Boettger T, Nyholm T, Karlsson M, Nunna C, Celi JC: Radiation therapy planning and simulation with magnetic resonance images. Proc SPIE 2008, 6918(69181C-1). [http://link.aip.org/link/PSISDG/v6918/i1/ p69181C/s1\&Agg=doi].

6. Stanescu T, Jans HS, Pervez N, Stavrev P, Fallone BG: A study on the magnetic resonance imaging (MRI)-based radiation treatment planning of intracranial lesions. Phys Med Biol 2008, 53(13):3579-3593. [http://www.ncbi.nlm.nih.gov/pubmed/18560047].

7. Wang C, Chao M, Lee L, Xing L: MRI-based treatment planning with electron density information mapped from $\mathrm{CT}$ images: a preliminary study. Technol Cancer Res Treat 2008, 7(5):341-348. [http://www.ncbi. nlm.nih.gov/pubmed/18783283].

8. Jonsson JH, Karlsson MG, Karlsson M, Nyholm T: Treatment planning using MRI data: an analysis of the dose calculation accuracy for different treatment regions. Radiat Oncol 2010, 5(62). [http://www. ncbi.nlm.nih.gov/pubmed/20591179].

9. Greer PB, Dowling JA, Lambert JA, Fripp J, Parker J, Denham JW, Wratten C, Capp A, Salvado O: A magnetic resonance imaging-based workflow for planning radiation therapy for prostate cancer. Med J Aust 2011, 194(4):24-27. [http://www.mja.com.au/public/issues/194_04_210211/ gre10325_fm.html].

10. Johansson A, Karlsson M, Nyholm T: CT substitute derived from MRI sequences with ultrashort echo time. Med Phys 2011, 38(5):2708-2714. [http://link.aip.org/link/MPHYA6/v38/i5/p2708/s1\&Agg=doi].

11. Dowling Ja, Lambert J, Parker J, Salvado O, Fripp J, Capp A, Wratten C, Denham JW, Greer PB: An atlas-based electron density mapping method for magnetic resonance imaging (MRI)-alone treatment planning and adaptive MRI-based prostate radiation therapy. Int J Radiat Oncol Biol Phys 2012, 83:e5-11. [http://www.ncbi.nlm.nih.gov/ pubmed/22330995].

12. Johansson A, Karlsson M, Yu J, Asklund T, Nyholm T: Voxel-wise uncertainty in CT substitute derived from MRI. Med Phys 2012, 39(6):3283-3290. [http://pdfserv.aip.org/MPHYA6/vol_39/iss_6/3283_1. pdf].

13. Hofmann M, Steinke F, Scheel V, Charpiat G, Farquhar J, Aschoff P, Brady M, Schölkopf B, Pichler BJ: MRI-based attenuation correction for PET/MRI: a novel approach combining pattern recognition and atlas registration. J Nucl Med: Official Publication, Soc Nucl Med 2008, 49(11):1875-1883. [http://www.ncbi.nlm.nih.gov/pubmed/18927326]

14. Hofmann M, Pichler B, Schölkopf B, Beyer T: Towards quantitative PET/MRI: a review of MR-based attenuation correction techniques. Eur J Nucl Med Mol Imaging 2009, 36(Suppl 1):93-104. [http://www.ncbi. nlm.nih.gov/pubmed/19104810].

15. Keereman V, Fierens Y, Broux T, Deene DeY, Lonneux M, Vandenberghe S: MRI-based attenuation correction for PET/MRI using ultrashort echo time sequences. J Nucl Med: Official Publication, Soc Nucl Med 2010, 51(5):812-818. [http://www.ncbi.nlm.nih.gov/pubmed/20439508].

16. Catana C, van der Kouwe A, Benner T, Michel CJ, Hamm M, Fenchel M, Fischl B, Rosen B, Schmand M, Sorensen AG: Toward implementing an MRI-based PET attenuation-correction method for neurologic studies on the MR-PET brain prototype. J Nucl Med: Official Publication, Soc Nucl Med 2010, 51(9):1431-1438. [http://www.ncbi.nlm.nih.gov/ pubmed/20810759].

17. Berker Y, Franke J, Salomon A, Palmowski M, Donker HCW, Temur Y, Mottaghy FM, Kuhl C, Izquierdo-Garcia D, Fayad Za, Kiessling F, Schulz V: MRI-based attenuation correction for hybrid PET/MRI systems: a 4-class tissue segmentation technique using a combined ultrashortecho-time/dixon MRI sequence. J Nucl Med 2012, 53(5):796-804. [http://jnm.snmjournals.org/cgi/doi/10.2967/jnumed.111.092577].

18. Bazalova M, Carrier JF, Beaulieu L, Verhaegen F: Dual-energy CT-based material extraction for tissue segmentation in Monte Carlo dose calculations. Phys Med Biol 2008, 53(9):2439-2456. [http://www.ncbi.nlm. nih.gov/pubmed/18421124]

19. Reichert ILH, Robson MD, Gatehouse PD, He T, Chappell KE, Holmes J, Girgis S, Bydder GM: Magnetic resonance imaging of cortical bone with ultrashort TE pulse sequences. Magn Reson Imaging 2005, 23(5):611-618. [http://www.ncbi.nlm.nih.gov/pubmed/16051035].

20. Stanisz GJ, Odrobina EE, Pun J, Escaravage M, Graham SJ, Bronskill MJ, Henkelman RM: T 1, T 2 relaxation and magnetization transfer in 
tissue at 3T. Magn Reson Med 2005, 54:507-512. [http://dx.doi.org/10 1002/mrm.20605].

21. Gold G, Han E, Stainsby J: Musculoskeletal, MRI at 3.0, T relaxation times and image contrast. Am J Roentgeno/ 2004, 183(8):343-351. [http://www.ajronline.org/content/183/2/343.short].

22. Robson MD, Gatehouse PD, Bydder M, Bydder GM: Magnetic resonance: an introduction to ultrashort TE (UTE) imaging. $J$ Comput Assist Tomogr 2003, 27(6):825-846. [http://www.ncbi.nlm.nih.gov/pubmed/14600447].

23. Robson $\mathrm{M}$, Bydder $\mathrm{G}$ : Clinical ultrashort echo time imaging of bone and other connective tissues. NMR Biomed 2006, 19:765-780. [http:// onlinelibrary.wiley.com/doi/10.1002/nbm.1100/pdf].

24. Rahmer J, Blume U, Börnert P: Selective 3D ultrashort TE imaging: comparison of "dual-echo" acquisition and magnetization preparation for improving short-T 2 contrast. Magn Reson Mater Phys Bio/ Med 2007, 20(2):83-92. [http://www.ncbi.nlm.nih.gov/pubmed/ 17354002].

25. Floca R: MatchPoint: on bridging the innovation gap between algorithmic research and clinical use in image registration. In World Congress on Medical Physics and Biomedical Engineering 2009, Munich, Germany Volume 25/4 of IFMBE Proceedings; 2009:1105-1108. [http://dx. doi.org/10.1007/978-3-642-03882-2 294].

26. Bouveyron C, Girard S, Schmid C: High dimensional discriminant analysis. Symp A Quarterly J Mod Foreign Literatures 2007, 36(14):1-9. [http://eprints.pascal-network.org/archive/00001047/]

27. Berge L, Bouveyron C, Girard S: HDclassif: An R package for model-based clustering and discriminant analysis of high-dimensional data. J Stat Softw 2012, 46(6):1-29.

28. R Development Core Team. R: A Language and Environment for Statistical Computing R Foundation for Statistical Computing R Foundation for Statistical Computing 2012. [http://www.r-project.org].

29. Schlegel W, Pastyr O, Bortfeld T, Becker G, Schad L, Gademann G, Lorenz WJ: Computer systems and mechanical tools for stereotactically guided conformation therapy with linear accelerators. Int $J$ Radiat Oncol Biol Phys 1992, 24(4):781-787.

30. Krämer M, Jäkel O, Haberer T, Kraft G, Schardt D, Weber U: Treatment planning for heavy-ion radiotherapy: physical beam model and dose optimization. Phys med biol 2000, 45:3299-3317. [http:// iopscience.iop.org/0031-9155/45/11/313].

31. Jäkel $\mathrm{O}$, Krämer $\mathrm{M}$, Karger $C P$, Debus J: Treatment planning for heavy ion radiotherapy: clinical implementation and application. Phys med biol 2001, 46:1101-1116. [http://www.ncbi.nlm.nih.gov/pubmed/ 20821103].

doi:10.1186/1748-717X-8-51

Cite this article as: Rank et al:: MRI-based treatment plan simulation and adaptation for ion radiotherapy using a classification-based approach Radiation Oncology 2013 8:51.

\section{Submit your next manuscript to BioMed Central and take full advantage of:}

- Convenient online submission

- Thorough peer review

- No space constraints or color figure charges

- Immediate publication on acceptance

- Inclusion in PubMed, CAS, Scopus and Google Scholar

- Research which is freely available for redistribution

Submit your manuscript at www.biomedcentral.com/submit
C Biomed Central 